\title{
Life Events and Depression in Children with Pervasive Developmental Disorders ${ }^{1}$
}

\author{
Mohammad Ghaziuddin, ${ }^{2}$ Norman Alessi, and John F. Greden \\ University of Michigan, Ann Arbor
}

To determine the role of life events in the occurrence of depression in children with pervasive developmental disorders (PDD), we compared 11 patients (DSM-III-R; 9 male; 2 female; $\mathrm{M}$ age: 11.0 years; $\mathrm{M}$ full-scale IQ: 75.3) with $P D D$ and depression, with an age- and sex-matched control group of patients with PDD without depression (DSM-III-R; 9 male; 2 female; M age: 9.8 years; $M$ full-scale IQ: 60.6). Information was collected about the occurrence of unpleasant life events in the 12 months prior to the onset of depression. Depressed children experienced significantly more life events in the 12 months prior to the onset of depression. Exit events such as bereavement were more common in the depressed group. Findings suggest that, as in the general population, significant life events, particularly those with a negative impact, may contribute to the occurrence of depression in children with PDD. Future studies should explore the role of both biologic factors and environmental stressors in the onset of depression in this population.

\section{INTRODUCTION}

Several studies have demonstrated an excess of certain types of stressors before the onset of clinical depression. Compared to general population, patients with depression experience roughly a threefold increase in these stressors before the onset of the illness (Brown \& Harris, 1989). These stressors, commonly referred to as life events, have been

${ }^{1}$ From the Department of Psychiatry, University of Michigan. The authors thank Deanna Miner, Barbara Shaw, Laurie McGovern and the staff of the Child Psychiatry Inpatient Unit for their support.

Address all correspondence to Mohammad Ghaziuddin, University of Michigan Medical Center, 1500 East Medical Center Drive, Ann Arbor, Michigan 48109-0390. 
shown to precede the onset of depression in a variety of groups of patients including children (Goodyer, Kolvin, \& Gatzanis, 1985), the elderly (Murphy, 1982), people with chronic illnesses, and those with developmental disabilities (DD). In the latter group, unpleasant life events have been shown to frequently precede psychiatric referral and admission (Ghaziuddin, 1988; Stack, Haldipur, \& Thompson, 1987). However, persons with $\mathrm{DD}$ and mental retardation constitute a heterogeneous group of individuals; therefore, it is important to investigate if discrete diagnostic subgroups with the DD population, such as those suffering from autism and other pervasive developmental disorders (PDD), also experience an excess of life events preceding the onset of psychiatric disorders such as depression.

To our knowledge, such an attempt has not yet been made. Few studies have addressed the issue of psychiatric comorbidity, especially depression, in the autistic/PDD population (Ghaziuddin, Tsai, \& Ghaziuddin, 1992). Evidence is only now emerging about the occurrence of depression in persons with autism/PDD. The original attention was drawn by case reports. Komoto, Usui, and Hirata (1984) described two adolescents with periodic mood swings resembling bipolar disorder; the mood swings responded to carbamazepine. Gillberg (1985) described a patient with Asperger syndrome, widely regarded as a variant of autism, who developed manic-depressive illness and who also had a family history of that disorder. In a case series of five adults with PDD, Clarke, Littlejohns, Corbett, and Joseph (1989) described a 23-year-old man with autistic disorder and major depression. Realmoto and August (1991) described three patients with autism and catatonia and suggested that autistic patients with comorbid psychiatric disorders such as bipolar disorder may be at increased risk for catatonia. Ghaziuddin and Tsai (1991) described the presentation of depressive illness in a 17-year-old boy with Down syndrome and autism. In a subsequent study (Ghaziuddin et al., 1992), the same authors also reported that in an outpatient sample of 68 children and adolescents with autism, $6(9 \%)$ patients suffered from an associated psychiatric disorder, and 3 of these 6 patients (50\%) were clinically depressed. Therefore, the available evidence strongly suggests that depression can occur in persons with autism/PDD. If this is correct, then chronic difficulties and life events would be of significance in the occurrence of this disorder in people with autism also, as in the rest of the population. In the present study, we examine the occurrence of life events in a group of depressed children with autism/PDD and compare them with a control group of autistic/PDD children without comorbid depression. 


\section{METHOD}

The study was conducted at the University of Michigan Child Psychiatry inpatient unit over a period of 36 months. This is a short-term 10bed tertiary referral center for children with psychiatric disorders staffed by two board-certified child psychiatrists; two child psychiatry fellows; and a multidisciplinary team consisting, among others, of speech therapists, teachers, social workers, and occupational therapists.

Diagnosis of pervasive developmental disorders was based on the DSM-III-R criteria (American Psychiatric Association, 1987). All patients suspected of suffering from PDD underwent a standard diagnostic protocol consisting of parent interviews; psychiatric observation of the child; psychological testing; educational assessment; and speech and language evaluation. The Autism Behavior Checklist (ABC, Arick, \& Almond, 1980) was also used to supplement clinical information. Patients who met less than the required 8 of the criteria on the symptom checklist of autistic disorder were labeled as suffering from pervasive developmental disorders not otherwise specified (PDDNOS).

Diagnosis of depression was based on the DSM-III-R criteria and included the categories of major depression, dysthymia, and depression not otherwise specified. Assessment was based on a checklist derived from the DSM-III-R. The Reiss scale (1990), a comprehensive measure for assessment of psychopathology in children with mental retardation and psychiatric disorders, was also used to support the clinical diagnosis. This is an observer-rated scale that takes into account the last 3 months of behavior and is completed with the help of direct caregivers. Except in one subject, the scale was completed in all those who were mentally retarded. Eventually, a "best estimate" diagnosis was reached within a week of admission, taking into account all the available information. Another child psychiatrist (N.E.) evaluated the available information and confirmed the diagnosis. Patients were excluded from analysis if agreement about the diagnosis of depression was not reached between the two psychiatrists (M.G. and N.E.). Structured interviews for the diagnosis of psychiatric disorders in this population were not attempted because patients were often not able to cooperate with such interviews (Young, O’Brian, Gutterman, \& Cohen, 1987).

Assessment of life events was done by investigators who were blind to the hypothesis and was based on the Life Events schedule (Goodyer et al., 1985). This schedule was completed with the help of the primary caregiver, taking all available information into context. The Life Events schedule has been used in studies exploring the role of life events and psychiatric disorders in children and adolescents. It is a comprehensive 
schedule that includes questions on a variety of areas of the child's life. Events are judged for their negative impact and for their independence, that is, the degree to which they appear to be beyond the control of the individual, such as bereavement, natural disasters, and so forth, and are not likely to be the result of illness. For the purpose of this study, only independent events were taken into account. The period under focus was 12 months prior to the onset of depression. Onset of symptoms was defined as any change in the patient's behavior suggesting depressive symptoms, such as crying spells, social withdrawal of a recent duration, increasing irritability, disturbance of sleep or of appetite, and the complaint of sadness in those who were able to volunteer an account of their illness. Anchor dates were used to clarify the time of onset of symptoms and the occurrence of life events.

Twelve patients with autism/PDD were eligible for the study. However, 1 patient, on whom agreement in diagnosis of depression could not be reached between the two child psychiatrists (M.G. and N.E.), was excluded. The final sample, therefore, consisted of 11 patients with autism/PDD with comorbid depression ( 9 male; $M$ full-scale IQ: $75.3 ; M$ age: 11 years; 4 autistic disorder, 7 PDDNOS). Six patients met the criteria for major depression, 3 of these with psychotic features, while the remaining 5 were categorized as suffering from depressive disorder not otherwise specified. Controls were chosen from a pool of nondepressed autistic/PDD patients admitted to the unit during the same index period, and matched on age, sex, and as close to the IQ as possible ( 9 male; $M$ full-scale IQ: $60.7 ; M$ age: 9.8 years; 4 autistic disorder; 7 PDDNOS). Five patients in the depressed group and 3 in the nondepressed group were mentally retarded (full-scale IQ < 70). Fisher's exact test (two-tailed) was used to determine the significance of the results (Table I).

\section{RESULTS}

Nine patients with depression (82\%) gave a history of having sustained recent unpleasant life events compared to $5(45 \%)$ in the nondepressed group. These events consisted of the change in group homes, change of education programs, family sickness, bereavement, and so forth. Five depressed patients gave a history of bereavement compared to one in the nondepressed group. The total mean life events score in the depressed group was significantly higher than that in the nondepressed group $(M=2.6 \pm 2.5$ vs. $0.7 \pm 1.0 ; . t=2.3$; $p=.03$; see Table I). 
Table I. Differences Between Depressed and Nondepressed Patients

\begin{tabular}{lccc}
\hline & Depressed group & Nondepressed group & $p$ \\
\hline Male:Female & $9: 2$ & $9: 2$ & \\
Age (years, $M \pm S D)$ & $11.0 \pm 2.5$ & $9.9 \pm 3.1$ & NS \\
Full-scale IQ $(M \pm S D$ & $75.3 \pm 19.6$ & $60.7 \pm 16.6$ & .07 \\
Mean Life Events score $(M \pm S D$ & $2.6 \pm 2.5$ & $0.7 \pm 1.0$ & .03 \\
\hline
\end{tabular}

Despite the presence of autism/PDD, it was possible to detect the occurrence of superimposed depression. A history of recent deterioration in overall functioning could be elicited in all the patients in the depressed group. The depressive symptoms consisted of a clear history of increasing social withdrawal, even when the patient's autistic traits were taken into account. In addition, evidence of loss of interest in activities, frequent crying spells, and disturbance of sleep and appetite, could be obtained. Eight patients presented with a history of having expressed suicidal thoughts that were of recent onset and did not constitute part of a repertoire of "autistic" behaviors. However, only 1 patient actually attempted to hurt herself. This was a 12-year old girl who was admitted after having cut across her face with a razor because she was feeling sad and depressed. She verbalized clearly her intent to kill herself. Another patient was convinced he was going to fall into the "dark hole of space" and die. It is important to note that his interest in scientific experiments took on a depressive flavor coinciding with the onset of depression and that his statements about falling into space did not seem to be the result of verbal rituals seen in some persons with autism.

\section{DISCUSSION}

The main finding of this study is that social factors and environmental stressors are important in the occurrence of depression in persons with autism/PDD. However, before drawing any conclusions, certain points need to be clarified. First, half of the sample had a fuil-scale IQ over 70. Since, most population studies of people with autism suggest that up to $75 \%$ of the patients are mentally retarded, the present sample may be considered 
rather unusual. While the higher full-scale IQ of the depressed group suggests that autistic/PDD children with higher IQ may be more likely to suffer from depression, the possibility that those with lower IQ do not report symptoms readily should also be considered. The second issue deals with the diagnosis of depression. The diagnosis of depression was made after careful consideration of all the available information and only after agreement was reached between both investigators (M.G. and N.A.). No structured interviews were conducted for the diagnosis of this disorder. This was because most of the patients were not able to cooperate with such interviews, an experience shared with other investigators who have tried to assess seriously disturbed children (McKenna et al., 1994; Young et al., 1987).

Despite these caveats, the findings underscore the fact that, even in people with autism/PDD, the role of stress and environmental factors in the occurrence of depression needs to be studied systematically. The depressed group gave a history of having experienced significantly more independent life events than the nondepressed group prior to the onset of their illness. This is particularly relevant because people with autism experience difficulty in adjusting to changes, and because all significant life events almost always result in some degree of change. In fact, this may be one manner in which people with developmental disabilities differ from the rest of the population in their response to life events. Apparently commonplace life events may provoke an unsettling and even an exaggerated response (Ghaziuddin, 1988), resulting in a certain degree of disorganization (Menolascino, 1983; Sovner, 1986). This emphasizes the necessity and importance of adequate preparation of people with autism before any changes, even if seemingly trivial, are planned.

A variety of life events were observed in the depressed group. Five $(45 \%)$ depressed patients gave a history of bereavement compared to 2 $(9 \%)$ in the nondepressed group. Several studies have stressed the role of bereavement in the occurrence of depression in the general population, in adults as well as in children. Association of behavioral disorder with bereavement has also been noted in persons with mental retardation (Emerson, 1977; Ghaziuddin, 1988). Other events which have been noted in persons with developmental disabilities in other studies (e.g., Ghaziuddin, 1988) were also found in the present sample: change of school, parental marital discord or illness, staff changes, and so forth. From a clinical standpoint, therefore, autistic and PDD children presenting to psychiatric services with a history of recent life events should be screened for depression. 
Although the role of life events in the occurrence of depression is generally acknowledged, the mechanisms that underlie the association are still not fully understood. In particular, the interaction between acute stressors and chronic adversity continues to be a topic of debate. Similarly, the role of genetic factors in the perception of life events has also not been fully clarified (McGuffin, Katz, \& Bebbington, 1988). Further, the significance of life events in the index episode and the subtype of depression has also not been fully explored (see Esquiga, Gutierrez, \& Lopez, 1987; Frank, Anderson, Reynolds, Ritenour, \& Kupfer, 1994; Ghaziuddin, Ghaziuddin, \& Stein, 1990; Post, Rubinow, \& Ballenger, 1986). So far as persons with autism/PDD are concerned, it is important to investigate if certain types of life events are more important here than in the general population. Another issue that needs to be studied is the role of mediating factors, such as level of IQ and verbal ability, that may contribute to the onset of depression in this group of patients. Moreover, the phenomenology of depression in this population needs to be studied systematically. Depression may be an underdiagnosed disorder in children with autism/PDD. Some of the social and communication deficits that occur in autism may mask the symptoms of depression and make the diagnosis difficult. Also, it is important to investigate if any particular subtype of autistic/PDD patients is more vulnerable to depression than others. Thus, future work should focus on the systematic characterization of depression in people with autism and incorporate measures of both psychosocial adversity and biologic vulnerability in the study of mood disorders in this population.

\section{REFERENCES}

American Psychiatric Association. (1987). Diagnostic and statistical manual for mental disorders (3rd ed., rev.). Washington, DC: Author.

Brown, G. W., Harris, T. O. (1989). Life events and illness. New York: Guilford.

Clarke, D. J., Littlejohns, C. S., Corbett, J. A., \& Joseph, S. (1989). Pervasive developmental disorders and psychoses in adult life. British Joumal of Psychiatry, 155, 692-699.

Esquiaga, E., Gutierrez, J. I. A., \& Lopez, A. J. (1987). Psychosocial factors and the episode number of depression. Journal of Affective Disorders, 12, 135-138.

Emerson, P. (1977). Covert grief reactions in mentally retarded clients. Mental Retardation, $15,46-47$.

Frank, E., Anderson, B., Reynolds, III C. F., Ritenour, A., \& Kupfer, D. J. (1994). Life events and the Research Diagnostic Criteria Endogenous Subtype. Archives of. General Psychiatry, 51, 519-524.

Ghaziuddin, M. (1988). Behavioral disorder in the mentally handicapped. The role of life events. British Joumal of Psychiatry, 152, 683-686.

Ghaziuddin, M., Ghaziuddin, N., \& Stein, G. (1990). Life events and the recurrence of depression. Canadian Joumal of Psychiatry, 35, 239-242. 
Ghaziuddin, M., \& Tsai, L. (1991). Depression in autistic disorder. British Jounal of Psychiatry, 159, 721-723.

Ghaziuddin, M., Tsai, L., Ghaziuddin, N. (1992). Comorbidity of autistic disorder in children and adolescents. European Child and Adolescent Psychiatry, 1(4): 209-213.

Gillberg, C. (1985). Asperger's syndrome and recurrent psychosis-A case study. Joumal of Autism and Developmental Disorders, 15, 389-397.

Goodyer, I., Kolvin, I., \& Gatzanis, S. (1985). Recent undesirable life events and psychiatric disorder in childhood and adolescence. British Journal of Psychiatry, 147, 517-523.

Komoto, J., Usui, S., \& Hirata, J. (1984). Infantile autism and affective disorder. Joumal of Autism and Developmental Disorders, 14, 81-84.

Krug, D. A., Arick, J., \& Almond, P. (1980). Behavior checklist for identifying severely handicapped individuals with high levels of autistic behavior. Joumal of Child Psychology and Psychiatry, 21, 221-229.

Menolascino, F. J. (1983). In F. J. Menolascino \& B. M. McCann (Eds.), Mental health and mental retardation: Bridging the gap (pp. 3-64). Baltimore, MD: University Park Press.

McGuffin, P., Katz, R., \& Bebbington, P. (1988). The Camberwell Collaborative Depression Study III: Depression and adversity in the relatives of depressed probands. British Journal of Psychiatry, 152, 775-782.

McKenna, K., Gordon, C., Lenane, M., Kaysen, D., Fahey, K., \& Rapoport, J. (1994). Looking for childhood-onset schizophrenia: the first 71 cases screened. Joumal of the American Academy of Child and Adolescent Psychiatry, 33, 636-644.

Murphy, E. (1982). The social origins of depression in old age. British Joumal of Psychiatry, $141,135-142$.

Post, R. M., Rubinow, D. R., \& Ballenger, J. C. (1986).Conditioning and sensitization in the longitudinal course of affective illness. British Joumal of Psychiatry, 149, 191-201.

Realmoto, G. M., \& August, G. J. (1991). Catatonia in autistic disorder: A sign of comorbidity or variable expression. Journal of Autism and Developmental Disorder, 21, 517-528.

Reiss, S. (1990). Reiss Scales for Children's Dual Diagnosis (Mental retardation and psychopathology). Worthington, OH: International Diagnostic Systems.

Sovner, R. (1986). Limiting factors in the use of DSM-III criteria with mentally ill/mentally retarded persons. Psychopharmacology Bulletin, 22, 1055-1059.

Stack, L. S., Haldipur, C. V., \& Thompson, M. (1987). Stressful life events and psychiatric hospitalization of mentally retarded patients. American Journal of Psychiatry, 144, 661-663

Young, J. G., O'Brian, J. D., Gutterman, E. M., \& Cohen, P. (1987). Research on the clinical interview. Journal of the American Academy of Child and Adolescent Psychiatry, 26, 613-620. 